\title{
The etiology and prevalence of urinary tract infection and asymptomatic bacteriuria in pregnant women in Iran: a systematic review and Meta-analysis
}

Milad Azami ${ }^{1,2}$, Zahra Jaafari ${ }^{3}$, Mansour Masoumi ${ }^{4}$, Masoumeh Shohani ${ }^{5}$, Gholamreza Badfar ${ }^{6}$, Leily Mahmudi ${ }^{7}$ and Shamsi Abbasalizadeh ${ }^{2 *}$

\begin{abstract}
Background: Urinary tract infection (UTI) is a common clinical problem in pregnant women. Bacteriuria in pregnancy without antibiotic treatment could result in complications. This study aims to investigate the etiology and prevalence of UTI and asymptomatic bacteriuria (ASB) in pregnant women in Iran.

Method: This meta-analysis follows the Preferred Reporting Items for Systematic Reviews and Meta-Analyses (PRISMA) guidelines. To avoid bias, all steps of the study were carried out independently by two researchers. We conducted a comprehensive search on all the related literature in national databases, including IranDoc, SID, Barakat Knowledge Network System, RICST, Magiran, Iranian National Library and international databases, including Scopus, Embase, Science Direct, PubMed/ Medline, Cochrane Library, Web of Sciences, EBSCO, as well as Google Scholar search engine until June 2018. After considering the inclusion/exclusion criteria and qualitative evaluation, studies were analyzed based on random effects model using Comprehensive Meta-Analysis Software Version 2.
\end{abstract}

Results: In 31 studies with a sample size of 20,309, the prevalence of ASB in pregnant Iranian women was estimated to be $8.7 \%$ (95\%Cl: $7.2-10.4)$. The lowest and highest prevalence of ASB were observed in the third trimester $(6.1 \%$ [95\%Cl: 2.1-16.4]) and first trimester (11.7\% [95\%Cl: 7.9-16.9]), respectively. Subgroup analysis of the prevalence of ASB based on geographical region $(P=0.002)$ and province $(P<0.001)$ was significant but for the quality of studies $(P=0.51)$ was not significant. In 17 studies including 48,731 pregnant women, the prevalence of UTI was estimated to be 9.8\% (95\%Cl: 7.6-12.5). The test for subgroup differences of prevalence of UTI for province $(P<0.001)$ was significant but for geographical region $(P=061)$ and quality of studies $(P=0.11)$ was not significant. Meta-regression model for the prevalence of UTI and ASB in pregnant women in Iran based on year of the studies was significant $(P<0.001)$. The most common microorganism involved in the etiology of UTI (61.6\% [95\%Cl: 51.6-70.7]) and ASB (63.22\% [95\%Cl: 51.2-73.8]) was E. coli.

Conclusion: UTI and ASB are prevalent in pregnant women in Iran. Therefore, UTI screening is essential in pregnant women. The most common microorganism involved in the etiology of UTI and ASB in pregnant women in Iran is E.coli.

Keywords: Etiology, Urinary tract infection, Asymptomatic bacteriuria, Pregnant women, Iran

\footnotetext{
* Correspondence: shamciabbasalizadeh@gmail.com

${ }^{2}$ Department of Obstetrics \& Gynecology, Women's Reproductive Health

Research Center, School of Medicine, Tabriz University of Medical Sciences,

Tabriz, Iran

Full list of author information is available at the end of the article
}

(c) The Author(s). 2019 Open Access This article is distributed under the terms of the Creative Commons Attribution 4.0 International License (http://creativecommons.org/licenses/by/4.0/), which permits unrestricted use, distribution, and reproduction in any medium, provided you give appropriate credit to the original author(s) and the source, provide a link to the Creative Commons license, and indicate if changes were made. The Creative Commons Public Domain Dedication waiver (http://creativecommons.org/publicdomain/zero/1.0/) applies to the data made available in this article, unless otherwise stated. 


\section{Background}

Urinary tract infection (UTI) is a common clinical problem that constitutes about $1-6 \%$ of medical referrals and includes urinary tract, bladder and kidney infections [1]. UTI may be symptomatic or asymptomatic, while asymptomatic bacteriuria (ASB) is of particular importance due to lack of any symptom $[2,3]$. UTI and its related complications cause about 150 million deaths per year around the world [4].

In pregnant women, physiological and anatomical changes in the urinary tract, as well as immune system changes during pregnancy increase the prevalence of ASB and in some cases lead to the symptomatic infection, resulting in serious risks for both mother and fetus. Increasing age, parity, diabetes, sickle cell anemia, history of UTI, urinary tract disorders and immune deficiency may increase the risk of UTI in pregnant women [5-7].

Bacteriuria in pregnancy without antibiotic treatment could result in complications such as preterm labor, pre-eclampsia, hypertension, pyelonephritis, anemia, amnionitis, low birth weight, neonatal deaths (stillbirths), bacteremia and toxic septicemia [8-10]. Treatment of bacteriuria in pregnancy reduces the risk of complications. Therefore, screening for early diagnosis and treatment of bacteriuria in women during pregnancy is necessary to prevent its complications [11].

The overall prevalence of bacteriuria in pregnant Iranian women was reported to be $2-41 \%$ [11-54]. Therefore, there is inconsistency in the results of studies. Thus, determining the prevalence of ABS, UTI and the most common pathogenic microorganisms involved in its creation is a valuable diagnostic capability in different countries.

Because of the inconsistency in different reports, reviewing various studies cannot be sufficient to achieve this goal. In systematic reviews, examining all related documents and combining them through meta-analysis provides a more complete picture of the dimensions of a problem [55-57].

This study aims to assess the prevalence of UTI, ASB and pathogens involved in bacteriuria among pregnant women in Iran.

\section{Methods}

\section{Study protocol}

This systematic review and meta-analysis follows the Preferred Reporting Items for Systematic Reviews and Meta-Analyses (PRISMA) guidelines [57]. To avoid bias, all steps of study were carried out independently by two researchers and in case of controversies, the problem was resolved by a third researcher.

\section{Inclusion and exclusion criteria}

Inclusion criteria according to PICO (Evidence-Based Medicine) [58] were as follows [1]: Population: The epidemiologic studies that investigated UTI, ASB and etiology among pregnant women [2]; Intervention: Urine culture for confirmed UTI and ASB [3]; Comparison: That can show the prevalence of UTI and ASB based on geographical region, province and trimester of pregnancy [4]; Outcome: Studies that estimated the UTI, ASB and etiology prevalence in pregnant women.

The exclusion criteria were: 1 . Non-random sampling; 2. Non-pregnant Iranian women; 3. Irrelevance with the subject of the research; 4. Incomplete information such as failing to report the prevalence; 5. Qualitative studies; 6 . Review articles, case reports and editorials; 7. Duplicates.

\section{Search strategy and study selection}

We conducted a comprehensive search on all English and Persian related literature in national databases, including Iranian Research Institute for Information Science and Technology (IranDoc) (https://irandoc.ac.ir), Scientific Information Database (SID) (http://www.sid.ir/), Barakat Knowledge Network System (http://health.barakatkns.com), Regional Information Center for Science and Technology (RICST) (http://en.ricest.ac.ir/), Magiran (http://www. magiran.com/), Iranian National Library (http://www.nlai.ir/ ) and international databases, including Scopus, PubMed/ Medline, Science Direct, Cochrane Library, Embase, Web of Sciences, EBSCO, as well as Google Scholar search engine until June 2018. We searched the articles using English MeSH keywords and Persian equivalents: "Pregnant", "Gestational", "Pregnancy", "Prenatal Care", "Urinary Tract Infection", "Bacteriuria", "Iran" and all possible combinations of keywords using "AND" and "OR" operators for English databases. In addition, the manual search was conducted to find more studies by screening the reference list of all articles included in the meta-analysis. PubMed combination search was as follows: ("Pregnant"[Title/Abstract] OR "Pregnancy"[Title/Abstract]) OR "Gestational"[Title/Abstract] OR "Prenatal Care"[Title/Abstract] AND ("Urinary Tract Infection"[Title/Abstract] OR "Bacteriuria"[Title/Abstract] AND "Iran"[Title/Abstract/Affiliation].

It is worth noting that 'High Sensitive Searching' was used in databases; in addition, the search was conducted by qualified researchers and experts in the field of database searching ("M. Azami" and "Z. Jaafari”).

\section{Quality assessment}

Authors assessed the quality of studies according to the modified Newcastle Ottawa Scale (NOS) for cross-sectional studies [59], which includes eight sections, and evaluated the selected articles from the selection, comparability, exposure assessment, and outcome. Points of $0-5,6-7$ and 8-10 were considered as low quality, moderate quality and high quality, respectively. A minimum score of 6 was considered as a criterion to include an article. 


\section{Data extraction}

The checklist was designed based on goals. This checklist included: authors, place, province, region, year of publication, year of study, study design, mean age, sample size, prevalence of UTI, ASB and microorganisms involved in bacteriuria. Sample size and prevalence of UTI and ASB for the first, second and third trimester of pregnancy were independently extracted by two researchers.

\section{Statistical analysis}

Binomial distribution formula was used to estimate the standard error for the prevalence of UTI, ASB and pathogens involved in bacteriuria. The heterogeneity of the studies was assessed using Cochran's Q test and $\mathrm{I}^{2}$ index, and interpreted as follows: 0-24\% may not be important, $25-49 \%$ indicates moderate heterogeneity, $50-75 \%$ indicates substantial heterogeneity and over $75 \%$ indicates considerable heterogeneity [60]. To combine data in high heterogeneity, we used the random effects model. To explore the potential sources of heterogeneity, subgroup analysis was preformed based on geographical region, province and trimester of pregnancy $[61,62]$. We used the meta-regression model for the prevalence of UTI and
ASB according to year of the study. Publication bias was measured by reviewing the funnel plots and through Begg and Egger's tests. Meta-analysis of data was performed using Comprehensive Meta-Analysis Software Version 2 and the significance level was considered less than 0.05 .

\section{Results}

\section{Search results}

In the systematic review, 520 potentially relevant articles were identified, and after screening the titles and abstracts, 260 studies were excluded because of being duplicate, and the full text of 260 possibly related articles was studied. After the evaluation of exclusion/inclusion criteria and the quality of articles, 42 eligible studies, published from 1995 to 2015, were included in meta-analysis (Fig. 1).

\section{Study characteristics}

42 eligible articles (17 studies for UTI and 31 studies for ASB) including 67,776 pregnant women were investigated. Mean and standard deviation (SD) for age was $26.47 \pm 5.47$ years. Other study characteristics are shown in Table 1.
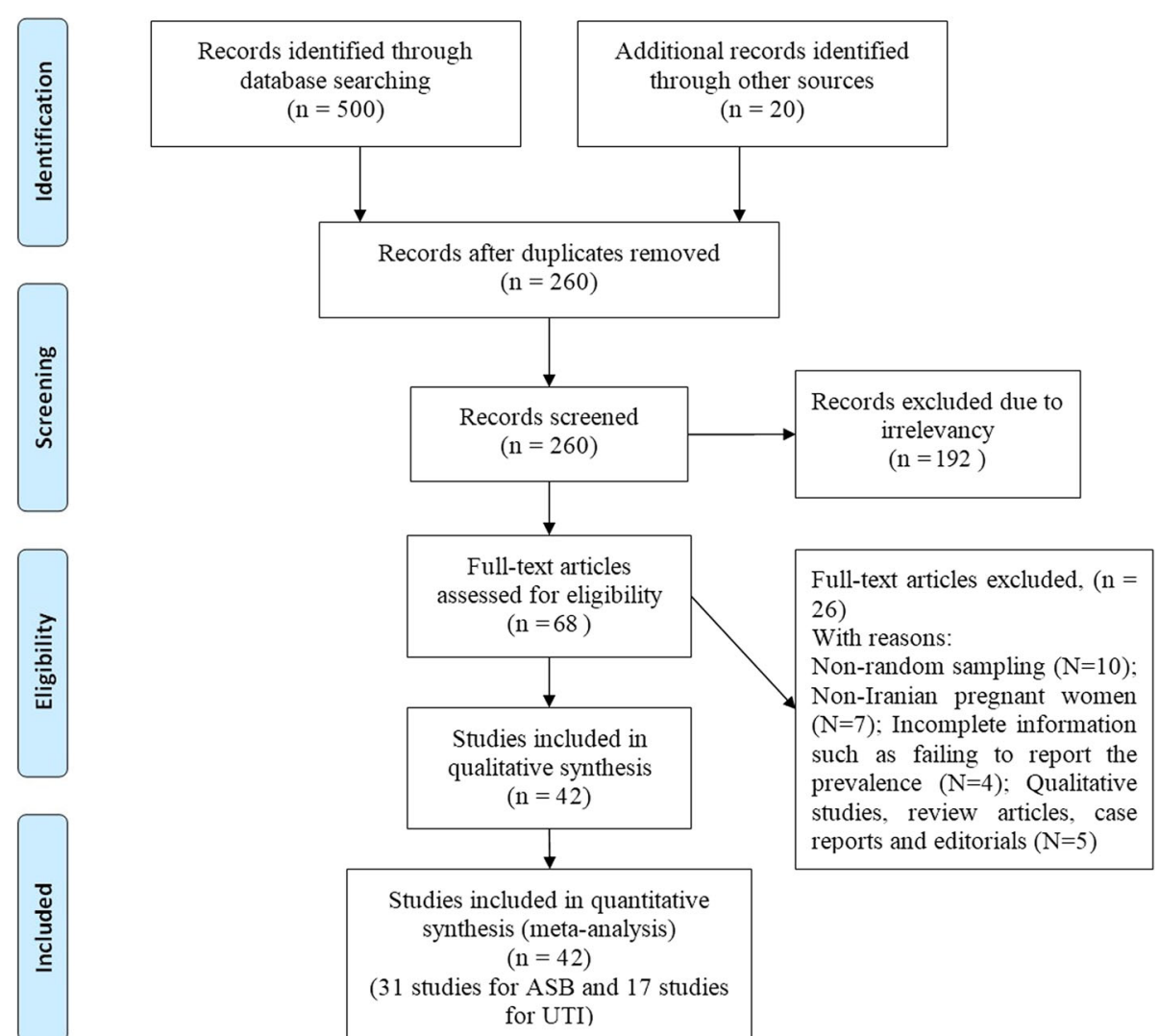

Studies included in quantitative synthesis (meta-analysis) $(n=42)$

(31 studies for ASB and 17 studies for UTI) 
Table 1 Characteristics of studies on asymptomatic bacteriuria and urinary tract infections among pregnant Iranian women

\begin{tabular}{|c|c|c|c|c|c|c|c|c|c|}
\hline Ref & $\begin{array}{l}\text { First author, } \\
\text { published year }\end{array}$ & Place & $\begin{array}{l}\text { Year of } \\
\text { study }\end{array}$ & $\begin{array}{l}\text { Sample } \\
\text { size }\end{array}$ & $\begin{array}{l}\left(\text { Mean } \pm S D^{a}\right) \\
\text { age }\end{array}$ & $\begin{array}{l}\text { Prevalence of } \\
\text { ASB }^{\mathrm{b}}\end{array}$ & $\begin{array}{l}\text { Prevalence of } \\
\text { UTI }^{\mathrm{C}}\end{array}$ & Test & $\begin{array}{l}\text { Most common } \\
\text { microorganisms }\end{array}$ \\
\hline [12] & Vejdani MH, 1998 & Tabriz & 1995 & 950 & & 10.5 & & Culture & E.coli ${ }^{d}$ \\
\hline [13] & Farajzadegan Z, 2008 & Isfahan & 2008 & 100 & $25.1 \pm 3.6$ & 2 & & Culture & \\
\hline [14] & Shirazi MH, 2007 & Hamadan & 2007 & 377 & & 10.1 & & Culture & E.coli \\
\hline [15] & Safari M, 2008 & Yasuj & 2006 & 715 & $25 \pm 5.3$ & & 6 & Culture & \\
\hline [16] & Mobsheri E, 2002 & Gorgan & 2000 & 900 & & 3.7 & & Culture & E.coli \\
\hline [17] & Kameli M, 2013 & $\begin{array}{l}\text { Torbat } \\
\text { Haidaria }\end{array}$ & 2013 & 1250 & & 10 & & Culture & Staphylococcus epidermidis \\
\hline [18] & Soofizadeh N, 2012 & Sanandaj & 2009 & 1500 & $28.4 \pm 6.1$ & 7.6 & & Culture & - \\
\hline [19] & Azizzadeh Sh, 1999 & Tehran & 1997 & 547 & & 12.7 & & Culture & E.coli \\
\hline [20] & Aaron H, 2008 & Kerman & 2007 & 323 & & 24.1 & & Culture & - \\
\hline [21] & Aghaee alamouti M, 2010 & Tehran & 2009 & 826 & & 8.7 & & Culture & - \\
\hline [22] & Yousofzadeh Sh, 1995 & Kashan & 1995 & 400 & & 4.7 & 7.5 & Culture & - \\
\hline [23] & Necohesh L, 2005 & Ghods & 2004 & 100 & & 14 & & Culture & E.coli \\
\hline [24] & Namazi A, 2012 & Guilan & 2008 & 710 & $27.48 \pm 6.0$ & 21.1 & & Culture & - \\
\hline [25] & Shojaee H, 2000 & Shahrekord & 1999 & 864 & & 4.3 & 8.8 & Culture & E.coli \\
\hline [26] & Zarganj Fard A, 2000 & Arak & 2000 & 1736 & & 6.3 & & Culture & E.coli \\
\hline [27] & Khorshidi A, 1997 & Kashan & 1996 & 350 & $24 \pm 5.5$ & 5.4 & & Culture & E.coli \\
\hline [28] & Hazhir S, 2007 & Tabriz & 2007 & 1100 & & 6.1 & & Culture & - \\
\hline [29] & Motaghi M, 2012 & Mashhad & 2008 & 150 & & 10.7 & & Culture & E.coli \\
\hline [30] & Dadkhah F, 2011 & Tehran & 2010 & 1246 & & 9 & & Culture & - \\
\hline [31] & Kalantar E, 2008 & Sanandaj & 2008 & 1505 & $28.4 \pm 4.6$ & 8.9 & & Culture & E.coli \\
\hline [32] & Kasraeian M, 2009 & Shiraz & 2007 & 389 & $26.3 \pm 4.2$ & 5.1 & & Culture & E.coli \\
\hline [33] & $\begin{array}{l}\text { Jazayeri Moghadas A, } \\
2009\end{array}$ & Semnan & 2007 & 297 & & 3.3 & & Culture & E.coli \\
\hline [34] & Alavi-Naini R, 2003 & Zahedan & 2002 & 490 & & 14.9 & & Culture & E.coli \\
\hline [34] & Alavi-Naini R, 2003 & Zahedan & 2002 & 478 & & 10.5 & & Culture & E.coli \\
\hline [34] & Alavi-Naini R, 2003 & Zahedan & 2002 & 463 & & 16.2 & & Culture & E.coli \\
\hline [35] & Rahmani E, 2012 & Kermanshah & 2011 & 500 & $27.4 \pm 5.8$ & & 19.8 & Culture & - \\
\hline [36] & Norouzzadeh M, 1997 & Karaj & 1997 & 300 & & & 22.7 & Culture & E.coli \\
\hline [37] & Mardanian F, 2004 & Isfahan & 2004 & 543 & & 10.4 & & Culture & - \\
\hline [37] & Mardanian F, 2004 & Isfahan & 2004 & 911 & & 9.9 & & Culture & - \\
\hline [37] & Mardanian F, 2004 & Isfahan & 2004 & 891 & & 2.0 & & Culture & - \\
\hline [38] & Fakhimi L, 2002 & Zanjan & 2002 & 1012 & & & 13 & Culture & - \\
\hline [39] & Azizi A, 2015 & Sanqor & 2001 & 3157 & $26.5 \pm 5.5$ & & 5.8 & Culture & - \\
\hline$[40]$ & Jalali M, 2014 & Karaj & 2013 & 180 & $26 \pm 5.8$ & & 19.8 & Culture & E.coli \\
\hline [41] & Zakeri Hamidi M, 2006 & Mazandaran & 2004 & 300 & & & 8.33 & Culture & - \\
\hline [42] & Shahhosseini Z, 2012 & Sari & 2009 & 428 & $24.5 \pm 4.8$ & & 2.8 & Culture & - \\
\hline [43] & Rahimkhani M, 2008 & Tehran & 2012 & 86 & $26.8 \pm 5.5$ & 29.1 & & Culture & $\begin{array}{l}\text { Staphylococcus } \\
\text { epidermidis }\end{array}$ \\
\hline [44] & Golestan M, 2011 & Yazd & 2008 & 5897 & & & 6.98 & Culture & - \\
\hline [45] & Rahmanian M, 2014 & Semnan & 2012 & 160 & $27.8 \pm 5.4$ & 5.6 & & Culture & - \\
\hline [46] & Shams MR, 2000 & Tehran & 1996 & 205 & & 6.8 & & Culture & - \\
\hline [47] & Alijahan R, 2014 & Ardabil & 2011 & 2496 & & & 9.7 & Culture & E.coli \\
\hline [48] & Saffar MJ, 2008 & Sari & 2002 & 5600 & & & 12.6 & Culture & E.coli \\
\hline
\end{tabular}


Table 1 Characteristics of studies on asymptomatic bacteriuria and urinary tract infections among pregnant Iranian women (Continued)

\begin{tabular}{|c|c|c|c|c|c|c|c|c|c|}
\hline$\overline{\text { Ref }}$ & $\begin{array}{l}\text { First author, } \\
\text { published year }\end{array}$ & Place & $\begin{array}{l}\text { Year of } \\
\text { study }\end{array}$ & $\begin{array}{l}\text { Sample } \\
\text { size }\end{array}$ & $\begin{array}{l}\left(\text { Mean } \pm S D^{a}\right) \\
\text { age }\end{array}$ & $\begin{array}{l}\text { Prevalence of } \\
\text { ASB }^{b}\end{array}$ & $\begin{array}{l}\text { Prevalence of } \\
\text { UTI }{ }^{\mathrm{C}}\end{array}$ & Test & $\begin{array}{l}\text { Most common } \\
\text { microorganisms }\end{array}$ \\
\hline [49] & Soleimani Zadeh L, 2004 & Bam & 2001 & 850 & $25.5 \pm 6.6$ & & 12.3 & Culture & - \\
\hline$[50]$ & Modars Sh, 1998 & Tehran & 1996 & 462 & & 10.8 & & Culture & E.coli \\
\hline [51] & Sharemi H, 2013 & Rasht & 2012 & 330 & $27.9 \pm 5.8$ & & 23.6 & Culture & - \\
\hline [52] & Sohrabi D, 2011 & Zanjan & 2007 & 3102 & & & 5.83 & Culture & - \\
\hline [53] & Amiri M, 2015 & Dezful & 2012 & 22,600 & & 10.5 & 5 & Culture & E.coli \\
\hline
\end{tabular}

${ }^{\mathrm{a}}$ Standard deviation; ${ }^{\mathrm{b}}$ Asymptomatic bacteriuria; ${ }^{\mathrm{c}}$ Urinary tract infections; ${ }^{\mathrm{d}}$ Escherichia coli

* Some studies estimated the prevalence of UTI or ASB for more than 1 year and also regions. Each data was considered separately because of assessing the slope of prevalence in the years and estimating which region is the highest or lowest

\section{Total prevalence of ASB and sensitivity analysis}

The heterogeneity rate for the prevalence of ASB was high $\left(\mathrm{I}^{2}=93.38, P<0.001\right)$. In 31 studies with a sample size of 20,309, the prevalence of ASB in pregnant Iranian women was estimated to be $8.7 \%$ (95\% CI:7.2-10.4) (Fig. 2). The lowest and highest prevalence of ASB were 2 and 29.1\% in the studies of Farajzadegan [13] and Rahimkhani [43], respectively (Fig. 2). Sensitivity analysis for the prevalence of
ASB in Fig. 3 shows that after removing a study at a time, the result is still robust.

\section{Subgroup analysis of the prevalence of ASB based on geographical region and province}

The lowest prevalence of ASB in pregnant women was estimated to be in the South of Iran (5.1\% [95\% CI: 3.3-7.8]) and Golestan province $(3.7 \%$ [95\% CI: 2.6-5.2]). The

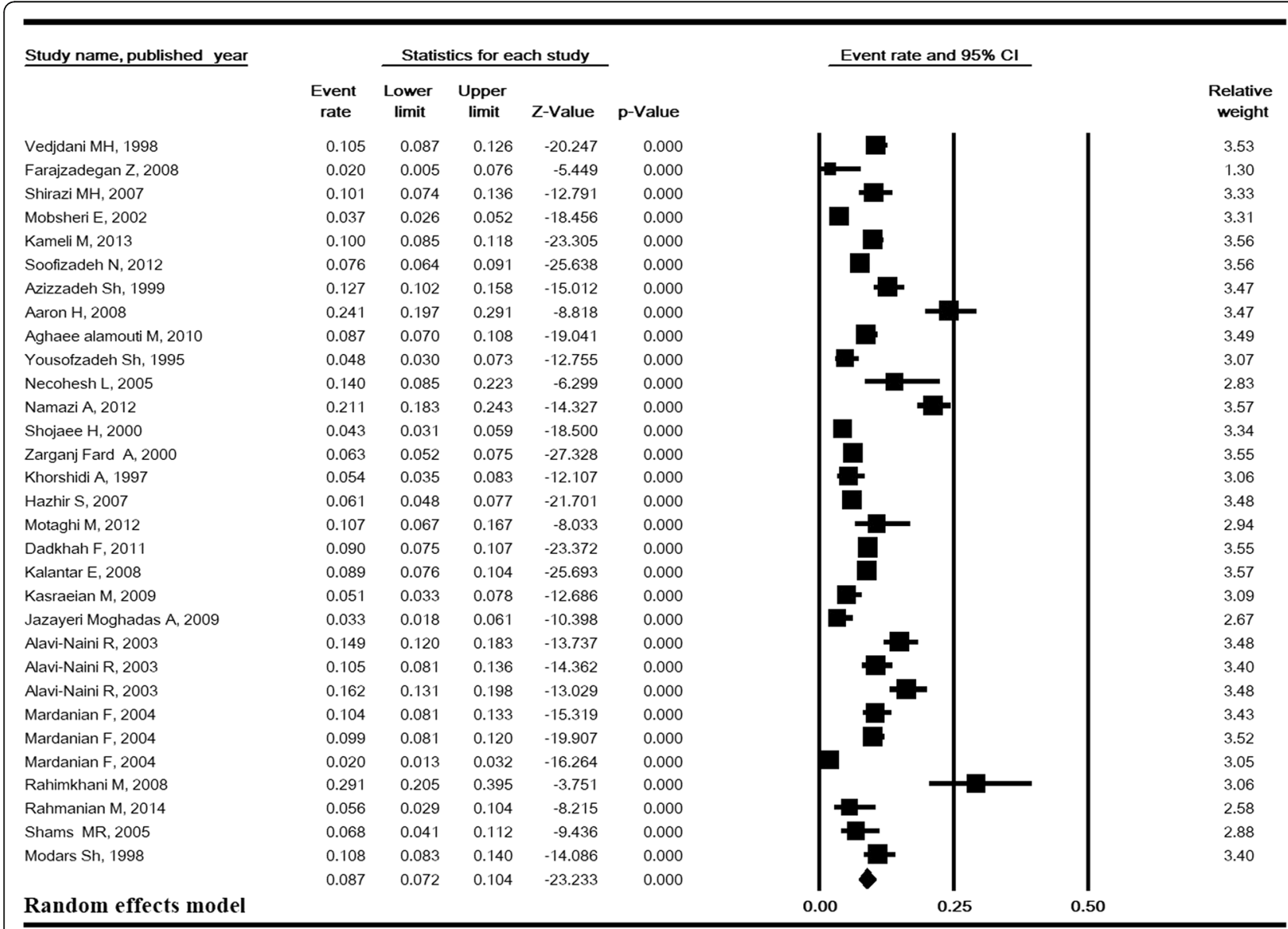

Meta Analysis

Fig. 2 Prevalence of asymptomatic bacteriuria in pregnant women in Iran 


\begin{tabular}{|c|c|c|c|c|c|c|c|}
\hline \multirow[t]{2}{*}{ Study name, published year } & \multirow[b]{2}{*}{ Point } & \multicolumn{3}{|c|}{ Statistics with study removed } & \multirow[b]{2}{*}{ p-Value } & Event rate $(95 \% \mathrm{Cl})$ & \multirow[t]{2}{*}{ with study removed } \\
\hline & & $\begin{array}{l}\text { Lower } \\
\text { limit }\end{array}$ & $\begin{array}{c}\text { Upper } \\
\text { limit }\end{array}$ & Z-Value & & & \\
\hline Vedjdani MH, 1998 & 0.086 & 0.071 & 0.104 & -22.291 & 0.000 & & \\
\hline Farajzadegan Z, 2008 & 0.089 & 0.074 & 0.106 & -22.978 & 0.000 & & \\
\hline Shirazi MH, 2007 & 0.086 & 0.072 & 0.104 & -22.644 & 0.000 & & \\
\hline Mobsheri E, 2002 & 0.090 & 0.075 & 0.107 & -23.173 & 0.000 & & \\
\hline Kameli M, 2013 & 0.086 & 0.071 & 0.104 & -22.114 & 0.000 & & \\
\hline Soofizadeh N, 2012 & 0.087 & 0.072 & 0.105 & -22.262 & 0.000 & & \\
\hline Azizzadeh Sh, 1999 & 0.086 & 0.071 & 0.103 & -22.664 & 0.000 & & \\
\hline Aaron H, 2008 & 0.084 & 0.070 & 0.100 & -24.673 & 0.000 & & \\
\hline Aghaee alamouti M, 2010 & 0.087 & 0.072 & 0.105 & -22.393 & 0.000 & & \\
\hline Yousofzadeh Sh, 1995 & 0.089 & 0.074 & 0.106 & -22.794 & 0.000 & & \\
\hline Necohesh L, 2005 & 0.086 & 0.071 & 0.103 & -22.993 & 0.000 & & \\
\hline Namazi A, 2012 & 0.084 & 0.071 & 0.100 & -25.241 & 0.000 & & \\
\hline Shojaee H, 2000 & 0.089 & 0.074 & 0.107 & -23.017 & 0.000 & & \\
\hline Zarganj Fard A, 2000 & 0.088 & 0.073 & 0.106 & -22.609 & 0.000 & & \\
\hline Khorshidi A, 1997 & 0.088 & 0.073 & 0.106 & -22.751 & 0.000 & & \\
\hline Hazhir S, 2007 & 0.088 & 0.073 & 0.106 & -22.666 & 0.000 & & \\
\hline Motaghi M, 2012 & 0.086 & 0.072 & 0.104 & -22.838 & 0.000 & & \\
\hline Dadkhah F, 2011 & 0.087 & 0.072 & 0.105 & -22.163 & 0.000 & & \\
\hline Kalantar E, 2008 & 0.087 & 0.072 & 0.105 & -22.046 & 0.000 & & \\
\hline Kasraeian M, 2009 & 0.088 & 0.074 & 0.106 & -22.769 & 0.000 & & \\
\hline Jazayeri Moghadas A, 2009 & 0.089 & 0.074 & 0.107 & -22.867 & 0.000 & & \\
\hline Alavi-Naini R, 2003 & 0.085 & 0.071 & 0.102 & -22.938 & 0.000 & & \\
\hline Alavi-Naini R, 2003 & 0.086 & 0.071 & 0.104 & -22.585 & 0.000 & & \\
\hline Alavi-Naini R, 2003 & 0.085 & 0.071 & 0.102 & -23.141 & 0.000 & & \\
\hline Mardanian F, 2004 & 0.086 & 0.071 & 0.104 & -22.540 & 0.000 & & \\
\hline Mardanian F, 2004 & 0.086 & 0.071 & 0.104 & -22.309 & 0.000 & & \\
\hline Mardanian F, 2004 & 0.091 & 0.076 & 0.108 & -23.504 & 0.000 & & \\
\hline Rahimkhani M, 2008 & 0.084 & 0.070 & 0.100 & -23.965 & 0.000 & & \\
\hline Rahmanian M, 2014 & 0.088 & 0.073 & 0.105 & -22.802 & 0.000 & & \\
\hline Shams MR, 2005 & 0.088 & 0.073 & 0.105 & -22.757 & 0.000 & & \\
\hline \multirow[t]{2}{*}{ Modars Sh, 1998} & 0.086 & 0.071 & 0.104 & -22.604 & 0.000 & & \\
\hline & 0.087 & 0.072 & 0.104 & -23.233 & 0.000 & & \\
\hline Random effects model & & & & & & 0.00 & 0.50 \\
\hline
\end{tabular}

Meta Analysis

Fig. 3 Sensitivity analysis for the prevalence of asymptomatic bacteriuria in pregnant women in Iran

highest prevalence of ASB in pregnant women was estimated to be in the East of Iran (13.9\% [95\% CI: 10.3-18.6]) and Kerman province (24.1\% [95\% CI: 19.7-29.1]). The test for subgroup differences for geographical region $(P=0.002)$ and province $(P<0.001)$ was significant (Table 2$)$.

\section{Subgroup analysis of the prevalence of ASB based on quality of studies}

The prevalence of ASB among pregnant women in terms of quality of studies based on NOS checklist was estimated to be 9.3\% [95\% CI: 7.1-12.1]) and 8.3\% [95\% CI: 6.4-10.6]) in moderate-quality and high-quality studies, respectively. No significant difference was found $(P=0.51)$ (Table 2$)$.

The prevalence of ASB based on trimester of pregnancy The lowest and highest prevalence of ASB were estimated in the third trimester (6.1\% [95\% CI: 2.1-16.4]) and first trimester (11.7\% [95\% CI: 7.9-16.9]), respectively (Fig. 4).

\section{Prevalence of UTI in pregnant women}

The heterogeneity rate for the prevalence of UTI was high $\left(\mathrm{I}^{2}=98.12 \%, P<0.001\right)$. In 17 studies including 48,731 pregnant Iranian women, the prevalence of UTI was estimated to be $9.8 \%$ (95\% CI: 7.6-12.5). The lowest prevalence was in the study of Shahhosseini (2.8\%) and the highest prevalence was in the study of Sharemi (23.6\%) (Fig. 5). Sensitivity analysis by removing a study at a time showed that the result for the prevalence of UTI was robust (Fig. 6).

\section{Subgroup analysis of the prevalence of UTI based on geographical region and province}

The prevalence of UTI among pregnant women in the South of Iran (7.2\% [95\% CI: 3.9-13.1]) and Khuzestan 
Table 2 subgroup analysis for the prevalence of asymptomatic bacteriuria and urinary tract infections in pregnant women in Iran

\begin{tabular}{|c|c|c|c|c|c|c|c|c|c|c|c|c|}
\hline \multirow[t]{3}{*}{ Variable } & \multicolumn{6}{|c|}{ Asymptomatic bacteriuria } & \multicolumn{6}{|c|}{ Urinary tract Infection } \\
\hline & \multirow{2}{*}{$\begin{array}{l}\text { Studies } \\
\left(N^{a}\right)\end{array}$} & \multirow{2}{*}{$\begin{array}{l}\text { Sample } \\
\text { (N) }\end{array}$} & \multicolumn{2}{|c|}{ Heterogeneity } & \multirow[t]{2}{*}{$95 \% \mathrm{Cl}^{\mathrm{b}}$} & \multirow{2}{*}{$\begin{array}{l}\text { Pooled } \\
\text { prevale } \\
\text { nce (\%) }\end{array}$} & \multirow{2}{*}{$\begin{array}{l}\text { Studies } \\
\text { (N) }\end{array}$} & \multirow{2}{*}{$\begin{array}{l}\text { Sample } \\
\text { (N) }\end{array}$} & \multicolumn{2}{|c|}{ Heterogeneity } & \multirow[t]{2}{*}{$95 \% \mathrm{Cl}$} & \multirow{2}{*}{$\begin{array}{l}\text { Pooled } \\
\text { prevalence } \\
(\%)\end{array}$} \\
\hline & & & $\overline{1^{2}}$ & $P$-Value & & & & & $\overline{1^{2}}$ & P-Value & & \\
\hline \multicolumn{13}{|l|}{ Region } \\
\hline Center & 17 & 9724 & 90.01 & $<0.001$ & $5.8-9.4$ & 7.4 & 6 & 11,575 & 96.42 & $<0.001$ & $6.7-13.8$ & 9.7 \\
\hline East & 6 & 3154 & 90.19 & $<0.001$ & $10.3-18.6$ & 13.9 & - & - & - & - & - & - \\
\hline North & 3 & 2560 & 98.08 & $<0.001$ & $3.9-22.1$ & 9.7 & 6 & 9334 & 94.77 & $<0.001$ & $8.2-15.6$ & 11.4 \\
\hline South & 1 & 389 & - & - & $3.3-7.8$ & 5.1 & 3 & 24,165 & 97.55 & $<0.001$ & $3.9-13.1$ & 7.2 \\
\hline West & 4 & 4482 & 68.68 & 0.023 & $6.6-9.6$ & 8.0 & 2 & 3657 & 99.04 & $<0.001$ & $3.1-32.4$ & 11.0 \\
\hline \multicolumn{7}{|c|}{ Test for subgroup differences: $Q=17.07, \mathrm{df}(\mathrm{Q})=4, P=0.002$} & \multicolumn{6}{|c|}{ Test for subgroup differences: $Q=1.77, \mathrm{df}(\mathrm{Q})=3, P=0.61$} \\
\hline \multicolumn{13}{|l|}{ Province } \\
\hline East Azarbaijan & 2 & 2050 & 92.25 & $<0.001$ & $4.7-13.5$ & 8.1 & 1 & 400 & - & - & $5.3-10.5$ & 7.5 \\
\hline Isfahan & 6 & 3195 & 91.29 & $<0.001$ & $3.1-8.8$ & 5.2 & - & - & - & - & - & - \\
\hline Hamedan & 1 & 377 & - & - & $7.4-13.6$ & 10.1 & - & - & - & - & - & - \\
\hline Golestan & 1 & 900 & - & - & $2.6-5.2$ & 3.7 & - & - & - & - & - & - \\
\hline Khorasan Razavi & 2 & 1400 & 0 & 0.78 & $8.6-11.8$ & 10.1 & - & - & - & - & - & - \\
\hline Kurdistan & 2 & 3005 & 40.25 & 0.19 & $7.1-9.6$ & 8.3 & - & - & - & - & - & - \\
\hline Tehran & 7 & 3472 & 85.23 & $<0.001$ & $8.8-15.3$ & 11.7 & - & - & - & - & - & - \\
\hline Kerman & 1 & 323 & - & - & $19.7-29.1$ & 24.1 & 1 & 850 & - & - & $10.3-14.7$ & 12.3 \\
\hline Chaharmahal and Bakhtiari & 1 & 864 & - & - & $4.7-13.5$ & 8.1 & 1 & 864 & - & - & $7.1-10.9$ & 8.8 \\
\hline Markazi & 1 & 1736 & - & - & $5.2-7.5$ & 6.3 & - & - & - & - & - & - \\
\hline Fars & 1 & 389 & - & - & $3.3-7.8$ & 5.1 & - & - & - & - & - & - \\
\hline Semnan & 2 & 457 & 26.81 & 0.24 & $2.5-7.1$ & 4.3 & - & - & - & - & - & - \\
\hline Guilan & 1 & 710 & - & - & $18.3-24.3$ & 21.1 & 1 & 330 & - & - & $19.3-28.5$ & 23.6 \\
\hline Sistan and Baluchestan & 3 & 1431 & 71.41 & 0.03 & $10.8-17.5$ & 13.8 & - & - & - & - & - & - \\
\hline Kohgiloyeh and Boyerahmad & - & - & - & - & - & - & 1 & 715 & - & - & $4.5-8.0$ & 6.0 \\
\hline Kermanshah & - & - & - & - & - & - & 2 & 3657 & 99.04 & $<0.001$ & $3.1-32.4$ & 11.0 \\
\hline Alborz & - & - & - & - & - & - & 2 & 480 & 0 & 0.45 & $18.2-25.6$ & 21.6 \\
\hline Zanjan & - & - & - & - & - & - & 2 & 4114 & 98.11 & $<0.001$ & $3.9-18.5$ & 8.8 \\
\hline Mazandaran & - & - & - & - & - & - & 3 & 6328 & 94.07 & $<0.001$ & $3.2-14.6$ & 7.0 \\
\hline Ardebil & - & - & - & - & - & - & 1 & 2496 & - & - & $8.6-10.9$ & 9.7 \\
\hline Yazd & - & - & - & - & - & - & 1 & 5897 & - & - & $6.4-7.7$ & 7.0 \\
\hline Khuzestan & - & - & - & - & - & - & 1 & 22,600 & - & - & $4.7-5.3$ & 5.0 \\
\hline
\end{tabular}

Test for subgroup differences: $\mathrm{Q}=289.16, \mathrm{df}(\mathrm{Q})=13, \mathrm{P}<0.001$

Test for subgroup differences: $Q=491.83, \mathrm{df}(\mathrm{Q})=11, P<0.001$

Quality

\begin{tabular}{|c|c|c|c|c|c|c|c|c|c|c|c|}
\hline High & 19 & 12,500 & 94.19 & $<0.001$ & $6.4-10.6$ & 8.3 & 11 & 45,355 & 98.42 & $<0.001$ & $6.4-11.5$ \\
\hline Moderate & 12 & 7809 & 92.25 & $<0.001$ & $7.1-12.1$ & 9.3 & 6 & 3376 & 93.11 & 0.001 & $8.7-17.6$ \\
\hline
\end{tabular}

Test for subgroup differences: $\mathrm{Q}=0.42, \mathrm{df}(\mathrm{Q})=1, \mathrm{P}=0.51$

Test for subgroup differences: $\mathrm{Q}=2.45, \mathrm{df}(\mathrm{Q})=1, \mathrm{P}=0.11$

${ }^{\mathrm{a}}$ Number; ${ }^{\mathrm{b}}$ Confidence interval

province (5\% [95\% CI: 4.7-5.3]) were the lowest and in the North of Iran (11.4\% [95\% CI: 8.2-15.6]) and Alborz province (21.6\% [95\% CI: 18.2-25.6]) were the highest. The test for subgroup differences for geographical region $(P=061)$ was not significant but for province $(P<0.001)$ was significant (Table 2).

\section{Subgroup analysis of the prevalence of UTI based on quality of studies}

The prevalence of UTI among pregnant women in terms of quality of studies based on NOS checklist was estimated to be $12.5 \%$ [95\% CI: 8.7-17.6]) and 8.6\% [95\% CI: $6.4-11.5])$ in moderate-quality and high-quality studies, 


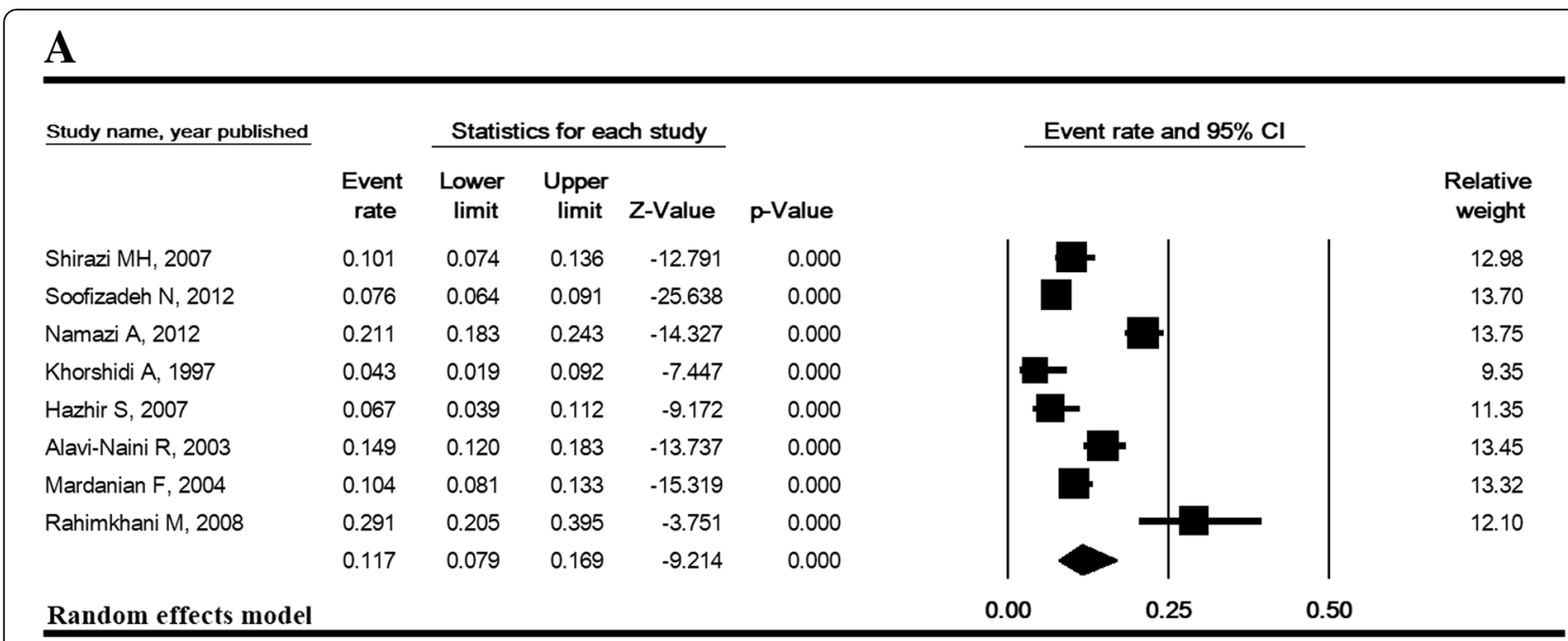

\section{Meta Analysis}

B

Study name, year published

$$
\begin{gathered}
\text { Event } \\
\text { rate }
\end{gathered}
$$

Farajzadegan Z, 2008

Khorshidi A, 1997

Hazhir S, 2007

Kasraeian M, 2009

Alavi-Naini R, 2003

Mardanian F, 2004

0.020

0.050

0.068

0.051

0.105

0.099

0.072
Statistics for each study

Lower Upper

limit limit Z-Value $p$-Value

$\begin{array}{lll}0.005 & 0.076 \quad-5.449\end{array}$

0.023

0.049

0.033

0.081

0.081

0.053
0.076

0.107

0.095

0.078

0.136

0.120

0.097
$-7.000$

$-14.333$

$-12.686$

$-14.362$

$-19.907$

$-15.172$
0.000

0.000

0.000

0.000

0.000

0.000

0.000
Event rate and $95 \% \mathrm{Cl}$

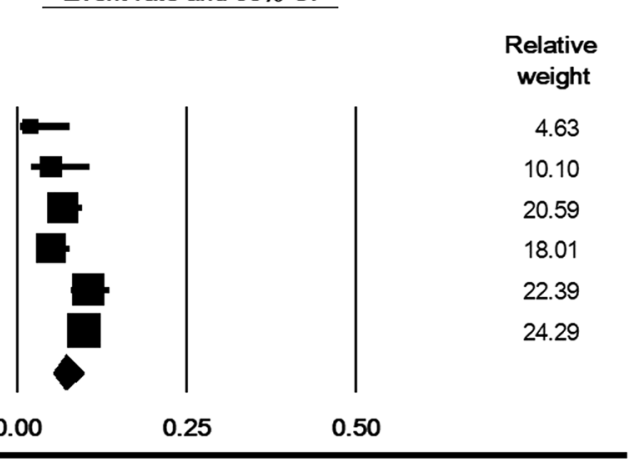

Random effects mode

\section{Meta Analysis}

C

Study name, year published

Statistics for each study

Event rate and $95 \% \mathrm{Cl}$

$\begin{array}{ccrrr}\begin{array}{c}\text { Event } \\ \text { rate }\end{array} & \begin{array}{c}\text { Lower } \\ \text { limit }\end{array} & \begin{array}{c}\text { Upper } \\ \text { limit }\end{array} & \text { Z-Value } & \text { p-Value } \\ 0.077 & 0.037 & 0.153 & -6.317 & 0.000 \\ 0.051 & 0.034 & 0.076 & -13.368 & 0.000 \\ 0.162 & 0.131 & 0.198 & -13.029 & 0.000 \\ 0.020 & 0.013 & 0.032 & -16.264 & 0.000 \\ 0.061 & 0.021 & 0.164 & -4.847 & 0.000\end{array}$

Khorshidi A, 1997

Hazhir S, 2007

Alavi-Naini R, 2003

Mardanian F, 2004

$$
0.061
$$

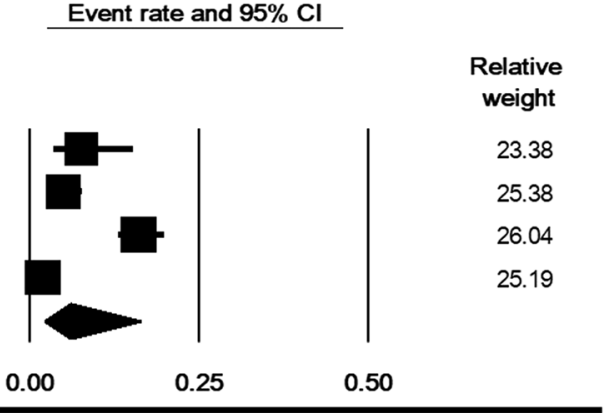

Random effects model

Meta Analysis

Fig. 4 Prevalence of asymptomatic bacteriuria in the first (a), second (b) and third (c) trimesters in Iran 


\begin{tabular}{|c|c|c|c|c|c|c|c|}
\hline \multirow[t]{2}{*}{ Study name, published year } & \multirow[b]{2}{*}{$\begin{array}{c}\text { Event } \\
\text { rate }\end{array}$} & \multicolumn{3}{|c|}{ Statistics for each study } & \multirow[b]{2}{*}{ p-Value } & \multirow[t]{2}{*}{ Event rate and $95 \% \mathrm{Cl}$} & \multirow[b]{2}{*}{$\begin{array}{l}\text { Relative } \\
\text { weight }\end{array}$} \\
\hline & & $\begin{array}{c}\text { Lower } \\
\text { limit }\end{array}$ & $\begin{array}{c}\text { Upper } \\
\text { limit }\end{array}$ & Z-Value & & & \\
\hline Safari M, 2008 & 0.060 & 0.045 & 0.080 & -17.473 & 0.000 & 1 & 5.77 \\
\hline Yousofzadeh Sh, 1995 & 0.075 & 0.053 & 0.105 & -13.234 & 0.000 & & 5.58 \\
\hline Shojaee H, 2000 & 0.088 & 0.071 & 0.109 & -19.471 & 0.000 & & 5.95 \\
\hline Rahmani E, 2012 & 0.198 & 0.165 & 0.235 & -12.464 & 0.000 & & 5.98 \\
\hline Norouzzadeh M, 1997 & 0.227 & 0.183 & 0.278 & -8.890 & 0.000 & & 5.87 \\
\hline Fakhimi L, 2002 & 0.130 & 0.111 & 0.152 & -20.337 & 0.000 & & 6.06 \\
\hline Azizi A, 2015 & 0.058 & 0.050 & 0.067 & -36.610 & 0.000 & & 6.11 \\
\hline Jalali M, 2014 & 0.198 & 0.146 & 0.263 & -7.479 & 0.000 & & 5.60 \\
\hline Zakeri Hamidi & 0.083 & 0.057 & 0.120 & -11.479 & 0.000 & & 5.46 \\
\hline Shahhosseini Z, 2012 & 0.028 & 0.016 & 0.049 & -12.106 & 0.000 & & 4.88 \\
\hline Golestan M, 2011 & 0.070 & 0.064 & 0.077 & -50.675 & 0.000 & & 6.17 \\
\hline Alijahan R, 2014 & 0.097 & 0.086 & 0.109 & -32.988 & 0.000 & & 6.13 \\
\hline Saffar MJ, 2008 & 0.126 & 0.118 & 0.135 & -48.097 & 0.000 & & 6.19 \\
\hline Soleimani Zadeh L, 2004 & 0.123 & 0.103 & 0.147 & -18.809 & 0.000 & & 6.01 \\
\hline Sharemi H, 2013 & 0.236 & 0.193 & 0.285 & -9.061 & 0.000 & & 5.91 \\
\hline Sohrabi D, 2011 & 0.058 & 0.051 & 0.067 & -36.306 & 0.000 & & 6.11 \\
\hline \multirow[t]{2}{*}{ Amiri M, 2015} & 0.050 & 0.047 & 0.053 & -96.473 & 0.000 & & 6.21 \\
\hline & 0.098 & 0.076 & 0.125 & -15.892 & 0.000 & & \\
\hline Random effects model & & & & & & 0.50 & \\
\hline
\end{tabular}

Meta Analysis

Fig. 5 Prevalence of urinary tract infection in pregnant women in Iran

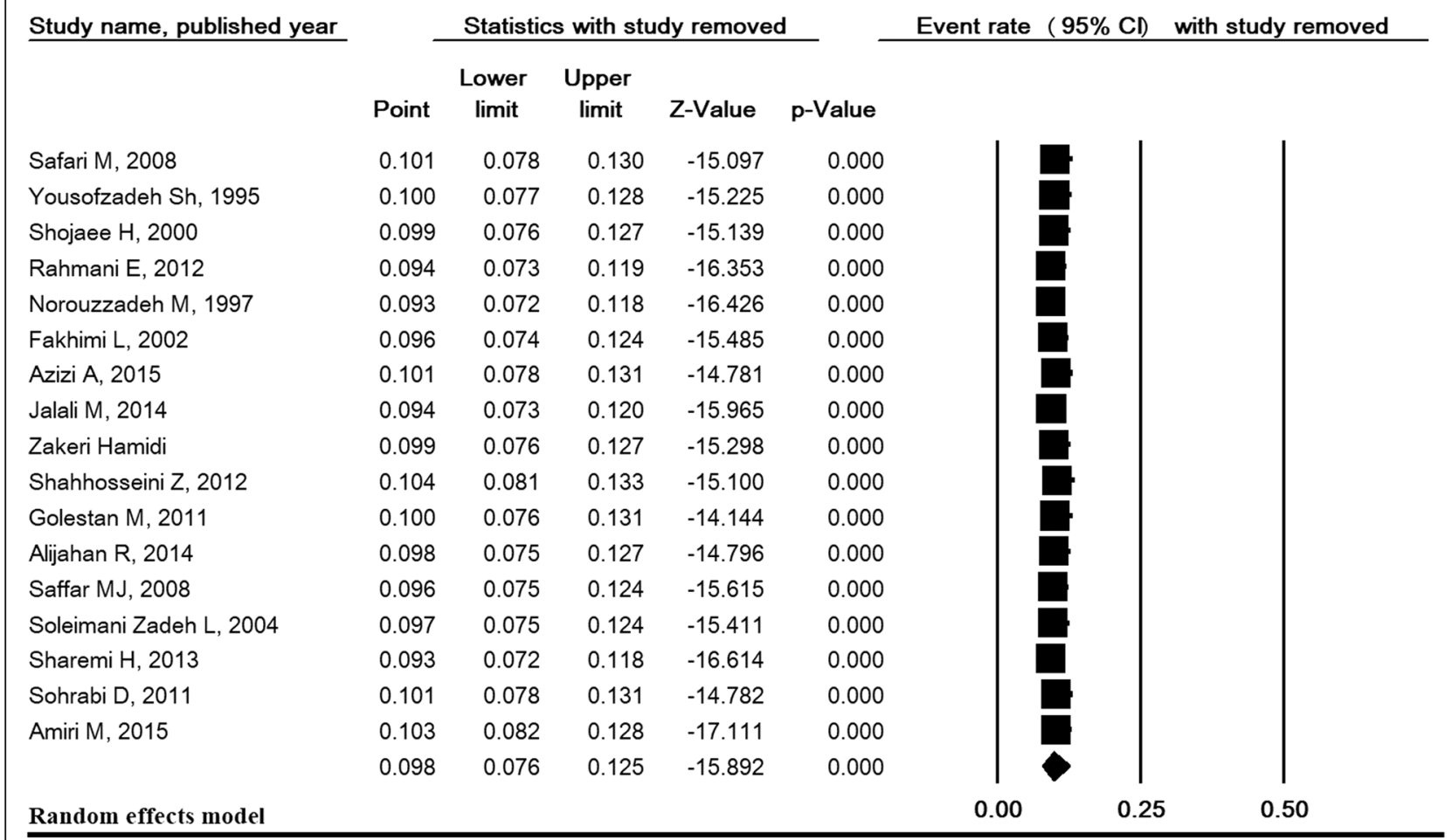

Meta Analysis

Fig. 6 Sensitivity analysis for the prevalence of urinary tract infection in pregnant women in Iran 


\begin{tabular}{|c|c|c|c|c|c|c|c|}
\hline \multirow[t]{2}{*}{ Study name, published year } & \multirow[b]{2}{*}{$\begin{array}{c}\text { Event } \\
\text { rate }\end{array}$} & \multicolumn{3}{|c|}{ Statistics for each study } & \multirow[b]{2}{*}{ p-Value } & \multicolumn{2}{|c|}{ Event rate and $95 \% \mathrm{Cl}$} \\
\hline & & $\begin{array}{c}\text { Lower } \\
\text { limit }\end{array}$ & $\begin{array}{c}\text { Upper } \\
\text { limit }\end{array}$ & Z-Value & & & $\begin{array}{c}\text { Relative } \\
\text { weight }\end{array}$ \\
\hline Safari M, 2008 & 0.060 & 0.045 & 0.080 & -17.473 & 0.000 & 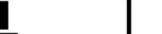 & 7.44 \\
\hline Yousofzadeh Sh, 1995 & 0.075 & 0.053 & 0.105 & -13.234 & 0.000 & & 7.11 \\
\hline Shojaee H, 2000 & 0.088 & 0.071 & 0.109 & -19.471 & 0.000 & & 7.78 \\
\hline Fakhimi L, 2002 & 0.130 & 0.111 & 0.152 & -20.337 & 0.000 & & 7.97 \\
\hline Azizi A, 2015 & 0.058 & 0.050 & 0.067 & -36.610 & 0.000 & & 8.08 \\
\hline Zakeri Hamidi M, 2006 & 0.083 & 0.057 & 0.120 & -11.479 & 0.000 & & 6.91 \\
\hline Shahhosseini Z, 2012 & 0.028 & 0.016 & 0.049 & -12.106 & 0.000 & & 5.95 \\
\hline Golestan M, 2011 & 0.070 & 0.064 & 0.077 & -50.675 & 0.000 & & 8.19 \\
\hline Alijahan R, 2014 & 0.097 & 0.086 & 0.109 & -32.988 & 0.000 & & 8.12 \\
\hline Saffar MJ, 2008 & 0.126 & 0.118 & 0.135 & -48.097 & 0.000 & & 8.23 \\
\hline Soleimani Zadeh L, 2004 & 0.123 & 0.103 & 0.147 & -18.809 & 0.000 & & 7.90 \\
\hline Sohrabi D, 2011 & 0.058 & 0.051 & 0.067 & -36.306 & 0.000 & & 8.07 \\
\hline \multirow[t]{2}{*}{ Amiri M, 2015} & 0.050 & 0.047 & 0.053 & -96.473 & 0.000 & & 8.26 \\
\hline & 0.076 & 0.060 & 0.097 & -18.572 & 0.000 & & \\
\hline Random effects model & & & & & & 0.25 & \\
\hline
\end{tabular}

\section{Meta Analysis}

\section{B}

Study name, published year

Vedjdani MH, 1998

Farajzadegan Z, 2008

Shirazi MH, 2007

Mobsheri E, 2002

Kameli M, 2013

Soofizadeh N, 2012

Azizzadeh Sh, 1999

Aghaee alamouti M, 2010

Yousofzadeh Sh, 1995

Necohesh L, 2005

Shojaee H, 2000

Zarganj Fard A, 2000

Khorshidi A, 1997

Hazhir S, 2007

Dadkhah F, 2011

Kalantar E, 2008

Kasraeian M, 2009

Jazayeri Moghadas A, 2009

Alavi-Naini R, 2003

Alavi-Naini R, 2003

Alavi-Naini R, 2003

Mardanian F, 2004

Mardanian F, 2004

Mardanian F, 2004

Rahmanian M, 2014

Shams MR, 2000

Modars Sh, 1998
Motaghi M, 2012

Statistics for each study

Event Lower Upper

rate limit limit Z-Value $p$-Value

$\begin{array}{lllll}0.105 & 0.087 & 0.126 & -20.247 & 0.000\end{array}$

$\begin{array}{lllll}0.020 & 0.005 & 0.076 & -5.449 & 0.000\end{array}$

$\begin{array}{llllll}0.101 & 0.074 & 0.136 & -12.791 & 0.000\end{array}$

$\begin{array}{lllll}0.037 & 0.026 & 0.052 & -18.456 & 0.000\end{array}$

$\begin{array}{llllll}0.100 & 0.085 & 0.118 & -23.305 & 0.000\end{array}$

$\begin{array}{lllll}0.076 & 0.064 & 0.091 & -25.638 & 0.000\end{array}$

$\begin{array}{llllll}0.127 & 0.102 & 0.158 & -15.012 & 0.000\end{array}$

$\begin{array}{llllll}0.087 & 0.070 & 0.108 & -19.041 & 0.000\end{array}$

$\begin{array}{llllll}0.048 & 0.030 & 0.073 & -12.755 & 0.000\end{array}$

$\begin{array}{llllll}0.140 & 0.085 & 0.223 & -6.299 & 0.000\end{array}$

$\begin{array}{llllll}0.043 & 0.031 & 0.059 & -18.500 & 0.000\end{array}$

$\begin{array}{llllll}0.063 & 0.052 & 0.075 & -27.328 & 0.000\end{array}$

$\begin{array}{llllll}0.054 & 0.035 & 0.083 & -12.107 & 0.000\end{array}$

$\begin{array}{llllll}0.061 & 0.048 & 0.077 & -21.701 & 0.000\end{array}$

$\begin{array}{llllll}0.107 & 0.067 & 0.167 & -8.033 & 0.000\end{array}$

$\begin{array}{llllll}0.090 & 0.075 & 0.107 & -23.372 & 0.000\end{array}$

$\begin{array}{llllll}0.089 & 0.076 & 0.104 & -25.693 & 0.000\end{array}$

$\begin{array}{llllll}0.051 & 0.033 & 0.078 & -12.686 & 0.000\end{array}$

$\begin{array}{llllll}0.033 & 0.018 & 0.061 & -10.398 & 0.000\end{array}$

$\begin{array}{llllll}0.149 & 0.120 & 0.183 & -13.737 & 0.000\end{array}$

$\begin{array}{llllll}0.105 & 0.081 & 0.136 & -14.362 & 0.000\end{array}$

$\begin{array}{llllll}0.162 & 0.131 & 0.198 & -13.029 & 0.000\end{array}$

$\begin{array}{llllll}0.104 & 0.081 & 0.133 & -15.319 & 0.000\end{array}$

$\begin{array}{llllll}0.099 & 0.081 & 0.120 & -19.907 & 0.000\end{array}$

$\begin{array}{llllll}0.020 & 0.013 & 0.032 & -16.264 & 0.000\end{array}$

$\begin{array}{llllll}0.056 & 0.029 & 0.104 & -8.215 & 0.000\end{array}$

$\begin{array}{llllll}0.068 & 0.041 & 0.112 & -9.436 & 0.000\end{array}$

$\begin{array}{lllll}0.108 & 0.083 & 0.140 & -14.086 & 0.000\end{array}$

$\begin{array}{lllll}0.078 & 0.067 & 0.090 & -29.821 & 0.000\end{array}$

Random effects model

\section{Meta Analysis}

Fig. 7 Prevalence of urinary tract infection (a) and asymptomatic bacteriuria (b) in pregnant women in Iran with deleted high prevalence reports (4 studies for prevalence of UTI: Rahmani, Norouzzadeh, Jalali and Sharemi and 3 studies for ASB: Namazi, Rahimkhani and Aaron)

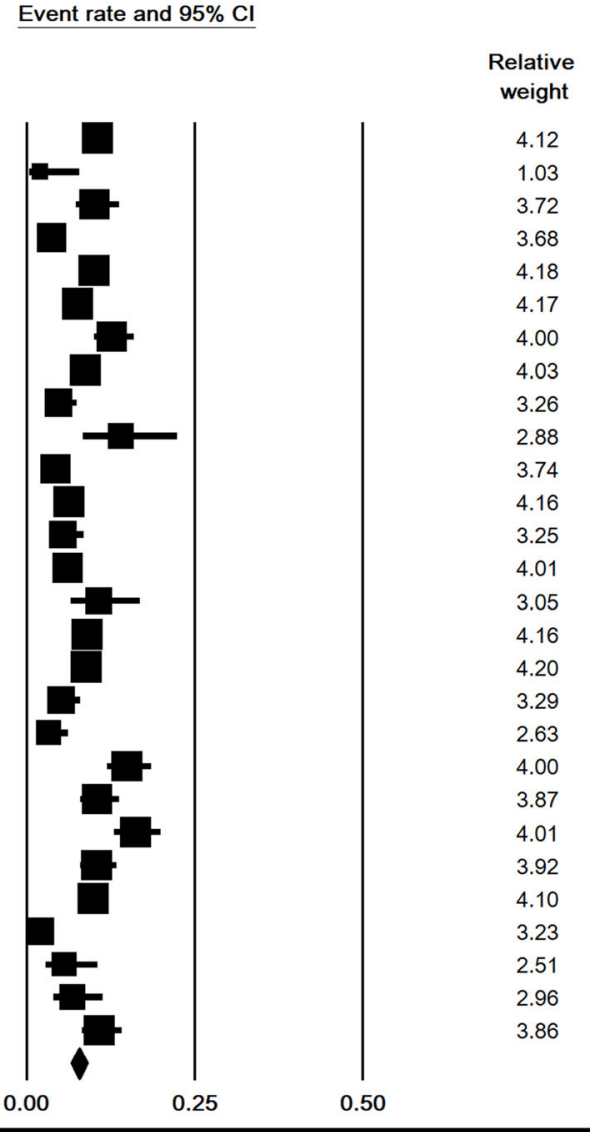


respectively. No significant difference was found $(P=0.11)$ (Table 2).

\section{Total prevalence of UTI and ASB with omission of high prevalence reports}

Four studies (Rahmani [35], Norouzzadeh [36], Jalali [40] and Sharemi [51]) for prevalence of UTI and three studies (Namazi [24], Aaron [20] and Rahimkhani [43]) for prevalence of ASB reported high prevalence. After omitting these studies, the prevalence of UTI and ASB was estimated to be $7.6 \%$ (95\% CI: $6.0-9.7$ ) and 7.8\% (95\% CI: 6.7-9.0), respectively (Fig. 7).

\section{Meta-regression}

Meta-regression model for the prevalence of UTI and ASB in pregnant women in Iran based on year of the studies was significant $(P<0.001$ for UTI and $P<0.001$ for ASB) (Fig. 8).

\section{The prevalence of microorganisms}

The most common microorganism involved in the etiology of UTI (61.6\% [95\% CI: 51.6-70.7]) and ASB (63.22\% [95\% CI: 51.2-73.8]) was E. coli. The lowest prevalence was Proteus with 2.6\% (95\% CI: 1.9-3.4) for UTI and 3.6\% (95\%CI: 2.0-6.3) for ASB (Table 3).

\section{Discussion}

The results of this meta-analysis indicated that the prevalence of ASB and UTI in pregnant Iranian women was 8.7 and 9.8\%, respectively. In subgroup analysis, geographic region, province and year of the studies can be a cause of heterogeneity between studies. The most common microorganism involved in the etiology of ASB and UTI in pregnant women in Iran was E. coli $(63.2$ and $61.6 \%$, respectively), while meta-regression model based on year of the studies for E. coli (for ASB) had a significantly decreasing trend. The healthcare structure for pregnant women is a state funded program running in Iran. This program included urine testing (urine culture test and urine analysis) in the first prenatal visit [12-15].

Prevalence of bacteriuria in pregnancy is affected by several factors such as multiple pregnancies, age, previous history of UTI, diabetes, urinary tract anatomic abnormalities, lack of personal hygiene and socioeconomic status $[63,64]$. In a systematic review conducted in Iran in 2015, ASB prevalence in pregnant women was reported to be $13 \%$ (95\% CI: 9-7) [65] after combining 20 articles (sample size: 15,108). In the present meta-analysis combining 31 studies with a sample size of 20,309 Iranian pregnant women, the prevalence of ASB was $8.7 \%$ (95\% CI: 7.2-10.4). The strengths of this study compared to previous published meta-analyses include bigger sample size, the use of cross-sectional studies, excluding studies with a non-randomized sample $[66,67]$ and removal of the duplicate articles that published the results more than once $[14,16,68,69]$. Each of the suggested factors can affect the final evaluation and accuracy of the prevalence while this was not considered in the previous meta-analyses [65].

The prevalence of ASB in pregnant Iranian women based on trimester of pregnancy shows that the highest prevalence occurs in the first trimester of pregnancy (11.7\% [95\% CI: 7.9-16.9]). Given that screening for UTI is done before the pregnancy and at 6-10 weeks of gestation in Iran, lack of care before pregnancy may increase the risk of UTI in pregnant women in the first trimester. However, trimester of pregnancy can be one of the causes of diverse prevalence of ASB in Iranian studies $(P=0.02)$.

There seem to be a geographical variation in the ASB prevalence, and we could not find the causes, but a possible reason might include differences in race (there is much racial diversity in Iran), socioeconomic factors, education, quality health care and women's health services communities [70, 71].

The prevalence of ASB among pregnant women in other countries, including India (7.3\%), Nigeria (24.7-

Table 3 The prevalence of Micro-Organisms in asymptomatic bacteriuria and urinary tract infections among Pregnant Iranian women

\begin{tabular}{|c|c|c|c|c|c|c|c|c|c|c|c|c|c|c|}
\hline \multirow{3}{*}{$\begin{array}{l}\text { Micro- } \\
\text { Organisms }\end{array}$} & \multicolumn{7}{|c|}{ Asymptomatic bacteriuria } & \multicolumn{7}{|c|}{ Urinary tract Infection } \\
\hline & \multirow{2}{*}{$\begin{array}{l}\text { Studies } \\
\left(N^{a}\right)\end{array}$} & \multirow{2}{*}{$\begin{array}{l}\text { Sample } \\
\text { (N) }\end{array}$} & \multicolumn{2}{|c|}{ Heterogeneity } & \multirow{2}{*}{$\begin{array}{l}\text { Pooled } \\
\text { prevalence (\%) } \\
{\left[95 \% \mathrm{Cl}^{\mathrm{b}}\right]}\end{array}$} & \multicolumn{2}{|c|}{$\overline{\text { Meta-regression }}$} & \multirow{2}{*}{$\begin{array}{l}\text { Studies } \\
(\mathrm{N})\end{array}$} & \multirow{2}{*}{$\begin{array}{l}\text { Sample } \\
\text { (N) }\end{array}$} & \multicolumn{2}{|c|}{ Heterogeneity } & \multirow{2}{*}{$\begin{array}{l}\text { Pooled } \\
\text { prevalence (\%) } \\
{[95 \% \mathrm{Cl}]}\end{array}$} & \multicolumn{2}{|c|}{ Meta-regression } \\
\hline & & & $\overline{1^{2}}$ & $P$-Value & & Trend & P-Value & & & $1^{2}$ & $P$-Value & & Trend & P-Value \\
\hline Escherichia coli & 18 & 1037 & 91.03 & $<0.001$ & $63.2[51.2-73.8]$ & $\mathrm{DES}^{\mathrm{C}}$ & $<0.001$ & 6 & 2255 & 93.34 & $<0.001$ & $61.6[51.6-70.7]$ & $\mathrm{ASC}^{\mathrm{C}}$ & 0.088 \\
\hline Staphylococcus & 15 & 902 & 88.09 & $<0.001$ & $19.6[12.7-29.2]$ & $\mathrm{ASC}^{\mathrm{d}}$ & $<0.001$ & 5 & 2187 & 58.99 & 0.045 & $9.9[7.7-12.6]$ & $\mathrm{DES}^{\mathrm{d}}$ & $<0.001$ \\
\hline Klebsiella & 12 & 783 & 42.86 & 0.057 & $6.3[4.2-9.4]$ & DES & 0.232 & 3 & 1448 & 90.05 & $<0.001$ & $13.9[7.3-25.0]$ & DES & 0.094 \\
\hline Streptococcus & 3 & 145 & 50.91 & 0.130 & $5.2[1.6-15.9]$ & ASC & 0.046 & - & - & - & - & - & - & - \\
\hline Enterococcus & 5 & 336 & 24.33 & 0.259 & $3.8[1.8-7.9]$ & DES & 0.165 & - & - & - & - & - & - & - \\
\hline Enterobacter & 8 & 608 & 29.33 & 0.194 & $6.4[4.1-9.7]$ & ASC & 0.578 & 2 & 776 & 61.51 & 0.107 & $7.6[4.3-13.1]$ & - & - \\
\hline Proteus & 6 & 356 & 0 & 0.766 & $3.6[2.0-6.3]$ & ASC & 0.383 & 3 & 1871 & 0 & 0.84 & 2.6 [1.9-3.4] & ASC & 0.820 \\
\hline
\end{tabular}

${ }^{\mathrm{a}}$ Number; ${ }^{\mathrm{b}}$ Confidence interval; ${ }^{\mathrm{c}}$ descending; ${ }^{\mathrm{d}}$ ascending 

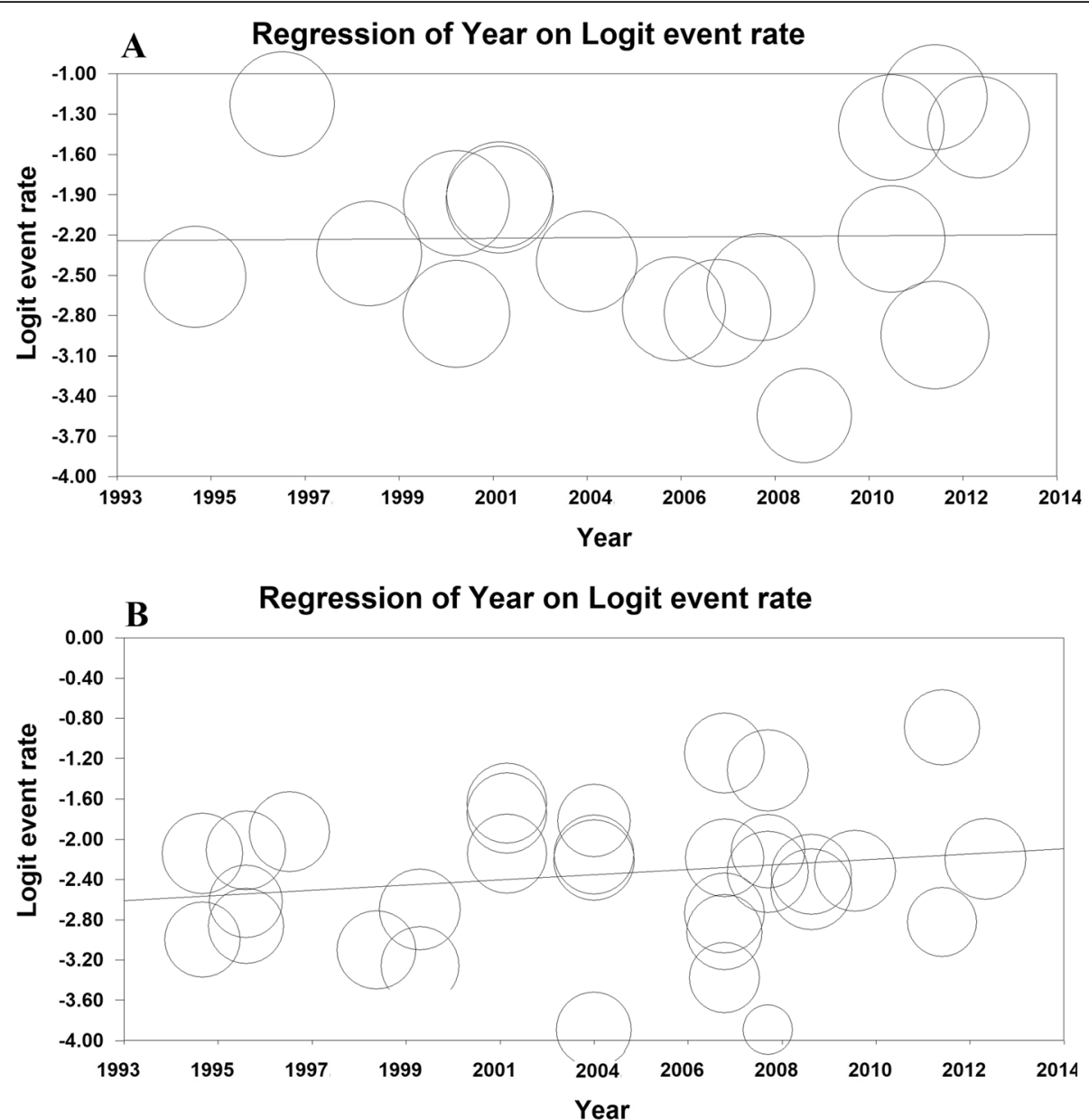

Fig. 8 Meta-regression model for the prevalence of urinary tract infection (a) and asymptomatic bacteriuria (b) in pregnant women in Iran based on year of the studies

45.3\%), Nepal (8.7\%) Bangladesh (10.2\%) and Ethiopia (21.2\%) was reported to be different [71-75].

The prevalence of UTI among young women is about $1-3 \%$ [76]. The results of this meta-analysis showed that the prevalence of UTI in pregnant Iranian women is high. Pregnant women prone to UTI are at risk for prematurity, preterm delivery, low birth weight, hypertension/pre-eclampsia, anemia, maternal and perinatal death associated with amnionitis $[75,76]$.

Studies show that the higher the education level, the lower the frequency of this problem. Hence, the need for education and awareness of pregnant women, especially in those with a lower education level, is necessary [18-20].

A common organism of ASB in pregnant women in Iran was E.coli (63.2\%). In other studies, the most common organism of ASB in women was E.coli [77]. E.coli is the underlying cause of ASB in $77 \%$ of sexually active young American women [78], 72\% of girls of school age [79], and $65-84 \%$ of pregnant women [80-83].
E. coli strains isolated from healthy women without symptoms may have a lower frequency of virulence factors, such as adhesions, specific lipopolysaccharide, toxins, mobility factors, and other proteins compared to strains isolated from symptomatic urinary tract infection $[84,85]$.

The aim of ASB treatment is to maintain sterile urine without causing toxicity in mother or fetus during pregnancy [82]. However, the best way to achieve this is not clear yet. In low-income countries, the situation is worse due to lack of information about the resistance to drugs used for UTI in pregnancy, drug costs and lack of access to information regarding the safety and efficacy of newer antibiotics [86].

In Iran, drug resistance in antibiotics used for UTI is a controversial topic. Different levels of antibiotic resistance and sensitivity has been reported in different studies. For example, in a study by Enaiat et al., high resistance of nalidixic acid, tetracycline and co-trimoxazole and low sensitivity of ampicillin, gentamicin and amikacin to E. coli has 
been reported in regard with bacteriuria in pregnant women [87]. However, in the study of Saffar, E.coli was highly sensitive to gentamicin and amikacin and less sensitive to trimethoprim-sulfamethoxazole and ampicillin. Therefore, considering the importance of empiric therapy for physicians, conducting a systematic review and meta-analysis to determine patterns of microbial resistance to drugs against UTI in Iran seems necessary.

\section{Limitations}

1. Lack of "AND" and "OR" operators support for a combined search in national databases.

2. Failure to investigate the prevalence of UTI based on trimester of pregnancy due to the limited number of studies.

3. Failure to investigate the prevalence of UTI based on factors such as multiple pregnancies, age, previous history of UTI, diabetes, anatomical abnormalities of urinary tract, lack of personal hygiene and socioeconomic status

\section{Conclusion}

UTI and ASB are highly common in pregnant women in Iran and the most common type of UTI is ASB. Therefore, it is recommended that urine culture be conducted as a part of routine tests for pregnant women. Moreover, pregnant women need to be provided with complete information about UTI complications during their pregnancy. The most common microorganisms involved in the etiology of ASB and UTI in pregnant women in Iran are E.coli and Staphylococcus. Since women and mothers' health is the foundation of the family and the public health, making better management decisions for prevention, screening and treatment of this problem is recommended.

\section{Abbreviations}

ASB: Asymptomatic Bacteriuria; IranDoc: Iranian Research Institute for Information Science and Technology; PRISMA: Preferred Reporting Items for Systematic Reviews and Meta-Analyses; RICST: Regional Information Center for Science and Technology; SID: Scientific Information Database; UTI: Urinary tract infection

\section{Acknowledgements}

The authors sincerely appreciate the Women's Reproduction Health Research Center of Tabriz University of Medical Sciences who helped us in accomplishing this research.

\section{Funding}

Not applicable.

\section{Availability of data and materials}

All data (literature) is available on Scopus, Embase, Science Direct, PubMed/ Medline, Cochrane Library, Web of Sciences, EBSCO, as well as Google Scholar.

\section{Authors' contributions}

MA, SHA and ZJ performed data extraction. MA and LM performed statistical analysis. MA and SHA wrote the manuscript. ZJ, GHB, MA, MSH and MM performed the literature search and quality evaluation. SHA, GHB, MSH, and MM revised the manuscript. All authors read and approved the final manuscript.

\section{Ethics approval and consent to participate}

Not applicable. Our manuscript is a systematic review of previous studies; therefore it does not report on or involve the use of any animal or human data or tissue during our study.

\section{Consent for publication}

Not applicable. Our manuscript is a systematic review of previous studies; therefore it does not contain any individual persons data obtained in our study.

\section{Competing interests}

The authors declare that they have no competing interests.

\section{Publisher's Note}

Springer Nature remains neutral with regard to jurisdictional claims in published maps and institutional affiliations.

\section{Author details}

${ }^{1}$ Faculty of Medicine, Ilam University of Medical Sciences, Ilam, Iran. ${ }^{2}$ Department of Obstetrics \& Gynecology, Women's Reproductive Health Research Center, School of Medicine, Tabriz University of Medical Sciences, Tabriz, Iran. ${ }^{3} \mathrm{HIV} / \mathrm{STI}$ Surveillance Research Center, and WHO Collaborating Center for HIV Surveillance, Institute for Futures Studies in Health, Kerman University of Medical Sciences, Kerman, Iran. ${ }^{4}$ Department of Urology, Faculty of Medicine, Ilam University of Medical Sciences, Ilam, Iran. ${ }^{5}$ Department of Nursing, Faculty of Nursing and Midwifery, Ilam University of Medical Sciences, Ilam, Iran. ${ }^{6}$ Department of Pediatrics, Behbahan Faculty of Medical Sciences, Behbahan, Iran. ${ }^{7}$ Faculty of Medicine, Dezful University of Medical Sciences, Dezful, Iran.

Received: 2 July 2018 Accepted: 31 March 2019

Published online: 30 May 2019

\section{References}

1. Gulfareen $\mathrm{H}$, Nishat $\mathrm{Z}$, AftabAfroze $M$, Ambreen $\mathrm{H}$. Risk factors of urinary tract infection in pregnancy. J Pak Med Assoc. 2010;60(3):213-6.

2. MacLean $A B$. Urinary tract infection in pregnancy. Int J Antimicrob Agents 2001:17(4):273-6.

3. Girishbabu RJ, Srikrishna R, Rameshs T. Asymptomatic bacteriuria in pregnancy. Int J Biol Med Res. 2011;2(3):740-2.

4. Totsika M, Moriel DG, Idris A, Rogers BA, Wurpel DJ, Phan MD, et al. Uropathogenic Escherichia coli mediated urinary tract infection. Curr Drug Targets. 2012;13(11):1386-99.

5. Counningham FG, Leveno KJ, Bloom SL, et al. Williams Obstetrics. 22nd ed. New York: McGraw_hill; 2005. p. 1095.

6. Giraldo PC, Araújo ED, Junior JE, Amaral RLGD, Passos MRL, Gonçalves AK. The prevalence of urogenital infections in pregnant women experiencing preterm and full-term labor. Infect Dis Obstetrics Gynecol. 2012;2012:1-4.

7. Raza S, Pandey S, Bhatt CP. Microbiological analysis of isolates in Kathmandu medical college teaching hospital, Kathmandu, Nepal. Kathmandu Univ Med J (KUMJ). 2011;9(36):295-7.

8. Glaser AP, Schaeffer AJ. Urinary Tract Infection and Bacteriuria in Pregnancy. Urol Clin North Am. 2015;42(4):547-60. https://doi.org/10.1016/j.ucl.2015.05. 004. Epub 2015 Aug 1

9. Bánhidy F, Acs N, Puhó EH, Czeizel AE. Maternal urinary tract infection and related drug treatments during pregnancy and risk of congenital abnormalities in the offspring. BJOG. 2006:113:1465-71.

10. Izadi B, Rostami-Far Z, Jalilian N, Khazaei S, Amiri A, Madani SH, Rostami-Far M. Urinary tract infection (UTI) as a risk factor of severe preeclampsia. Glob J Health Sci. 2016;8(11):54364.

11. Smaill F, Vazquez JC. Antibiotics for asymptomatic bacteriuria in pregnancy. Cochrane Database Syst Rev. 2015;8:CD000490.

12. Vejdani MH, Gourabchi H, Nahaie MRSM. Study of asymptomatic bacteriuria in pregnant women and antibiotic-sensitivity of isolated bacteria in Tabriz 1996. J Kerman Uni Med Sci. 1998;5(4):165-70. 
13. Farajzadegan Z, Mirmoghtadaee P, Mehrabian F. Screening of asymptomatic bacteriuria:urinalysis or urine culture? Which one is more cost- effective? J Isfahan Med School. 2008;26(89):119-26.

14. Shirazi MH, Ranjbar R, Daneshyar E, Sadeghian S, Sadeghifard N. Urinary tract pathogens in asymptomatic bacteriuria and their antimicrobial resistance in pregnant women in Hamedan. IJIDTM J. 2007;12(36):53-8.

15. Safari M, Yazdanpanah B, Yazdanpanah S. High risk pregnancy and some of related factors in women who Refered to Vasouj health and medical centers. Sci J Hamadan Nurs Midwifery Fac. 2008;16(2):18-28.

16. Mobsheri E, Tabbraei A, Ghaemei E, Mojerloo M, Vakili MA, Dastforooshan M, et al. Distribution of asymptomatic bacteriuria in pregnant women referring to Dezyani Hospital in Gorgan. J Gorgan Uni Med Sci. 2002;4(1):42-6.

17. Kameli M, Badiee M, Rafiee M. Prevalence of asymptomatic bacteriuria and its treatment in pregnant women referred to health centers of Torbat Haydarieh in 2013. J Torbat Heydariyeh Uni Med Sci. 2013;1(3):58-64.

18. Soofizadeh N, Farhadifar F, Kalantar E, Shahsavari S. Evaluating the prevalence of asymptomatic urinary tract infection and Anemia in pregnant women in first trimester. IJOGI. 2012;15(8):11-6.

19. Azizzadeh Sh, Ashtiani M, Baygi D. Evaluate the frequency of asymptomatic bacteriuria in pregnant women and diagnostic value of urine analysis in screening with its during 1999. [dissertation]. Tehran: Tehran Univ Med Sci; 1999.

20. Aaron $H$, Salari D. The prevalence of asymptomatic bacteriuria in pregnant women referred to Afzalipour haspital in Kerman. [Dissertation]. Kerman: Kerman Univ Med Sci; 2008.

21. Aghaee alamouti M, Moheit M. The prevalence of asymptomatic bacteriuria and social factors that influence the prevalence among women referred to hospitals in Tehran. [Dissertation]. Tehran: Islamic Azad Univ Med Sci; 2010.

22. Yousofzadeh Sh. Determine the prevalence of asymptomatic and symptomatic urinary tract infection in pregnant women referred to prenatal care in Kashan. [Dissertation]. Kashan: Kashan Univ Med Sci; 1995.

23. Necohesh $L$, Karimi MR. The Survey of bacterial agents of rinary tract infection among pregnant women in Ghods city in 2004-2005. [Dissertation]. Tehran: Islamic Azad Univ Med Sci; 2005.

24. Namazi A, Alizadeh S. The frequency of bacteriuria and its relationship with demographic characters in pregnant women of Gilan, 2009-11. J Kermanshah Univ Med Sci. 2012;16(3):263-8.

25. Shojaee $\mathrm{H}$. Microbiologic evaluation of bacteriuria in pregnant women in Shahrekord 1999. Pejouhandeh Q Res J. 2000;5(19):337-43.

26. Zarganj Fard A. The prevalence of asymptomatic bacteriuria in pregnant women who were referred to health centers of Arak city from November 1998 to March 1999. Arak Univ Med Sci (Rahavard Danesh). 2000;3(10):21-4.

27. Khorshidi A, Khakbazan S, Mousavi SGA, Hoshyaran A. Epidemiologic study of urinary infections without clinical symptoms among pregnant women: Diagnostic values of Nitrate among subjects referred to the Dr.Shabikhani Hospital, 1996. KAUMS Journal (FEYZ). 1997;1(3):53-60.

28. Hazhir S. Asymptomatic bacteriuria in pregnant women. Urol J. 2007;4(1): 24-7.

29. Motaghi M, Sadegh-Al-Hosseini A, Yaghoubi MA. Assessment of the risk factors of asymptomatic bacteriuria among pregnant and non-pregnant married women. Zahedan J Res Med Sci (ZJRMS). 2012;13(suppl 1):4.

30. Dadkhah F, Kashanian M, Noori M. A Comparison between Diagnostic Value of Pyuria and Bacteriuria in Asymptomatic Bacteriuria in Pregnant Women. IJOGI. 2011;14(1):48-53.

31. Kalantar E, Fariba F, Bahram N. Asymptomatic bacteriuria among pregnant women referred to outpatient clinics in Sanandaj, Iran. Int Braz J Urol. 2008; 34(6):699-704

32. Kasraeian M, Asadi N, Ghaffarpasand F. Prevalence of asymptomatic bacteriuria among pregnant women in shiraz, Iran. Saudi Med J. 2009;30(7): 917-20.

33. Jazayeri Moghadas A, Irajian G. Asymptomatic urinary tract infection in pregnant women. Iran J Pathol. 2009;4(3):105-8.

34. Alavi-Naini R, Sharifi-Mood B, Metanat M. The prevalence and drug resistance of a asymptomatic bacteriuria in pregnant women referring to zahedan ghods hospital. IJOGI. 2003;6(1):58-63.

35. Rahmani E, Karami A, Khalili M, Hasani S. A survey on the urinary tract infection (UTI) prevalence and its consequences in pregnant women referring to health centers in Kermanshah in 2011. J Clin Res Paramed Sci. 2012;1(4):31-8.
36. Norouzzadeh M, Sultandalal MM. Evaluation of Urinary Tract Infection in pregnant women admitted to Kasra hospital in Karaj. . [Dissertation]. Tehran: Islamic Azad Univ Med Sci; 1997.

37. Mardanian F, Atri S, Kian Pour M, Soleimani B, E Arab. Urinary tract infection in pregnancy. J Isfahan Med Sch. 2004;22(74-75):109-14.

38. Fakhimi L, Sohrabi D, Mousavi Nasab N, Fekri SH. Evaluate the frequency and risk factors for PROM in pregnant women referred off to hospital Valiasser in 2002. [Dissertation]. Zanjan: Zanjan Univ Med Sci; 2002.

39. Azizi A. The prevalence of the causes of high-risk pregnancies in pregnant women of Sonqor city, 2011. IJOGI. 2015;18(153):10-9.

40. Jalali M, Shamsi N, Roozbehani N, Kabir K. Prevalence of urinary tract infection and some factors affected in pregnant women in Iran Karaj City 2013. Middle East J Sci Res. 2014;20(7):781-5.

41. Zakeri Hamidi M. Urinary tract infections in pregnant women with bacterial vaginosis. Physician Lab Mon. 2006;2:47-48.

42. Shahhosseini Z, Esmaeeli Douki Z, Vaezzadeh N, Keshavarz Z. Maternal depression and preterm delivery. World Appl Sci J. 2012;18(1):15-20.

43. Rahimkhani M, Khavari-Daneshvar $H$, Sharifian R. Asymptomatic bacteriuria and pyuria in pregnancy. Acta Medica Iranica. 2008;46(5):409-12 B.

44. Golestan M, Akhavan Karbasi S, Fallah R. Prevalence and risk factors for low birth weight in Yazd, Iran. Singapore Med J. 2011:52(10):730-3.

45. Rahmanian M, Ghorbani R, Mirza Aghaie MR, Pourazizi M. Association of asymptomatic bacteriuria and preeclampsia in pregnant women. IJOGI. 2014;17(122):1-7.

46. Shams MR, Ghafarnezhad M. Prevalence and risk factors of asymptomatic bacteriuria in pregnancy. Tehran Univ Med J. 2000;58(2):76-9.

47. Alijahan R, Pourfarzi F, Salimi S, Tahmasebi P, Moradi S, Hazrati S. Prevalence of urinary tract infection and associated risk factors in pregnant women. Iran South Med J. 2014;17(4):676-86.

48. Saffar MJ, Enayti AA, Abdolla IA, Razai MS, Saffar H. Antibacterial susceptibility of uropathogens in 3 hospitals, sari, Islamic Republic of Iran, 2002-2003. East Mediterr Health J. 2008;14(3):556-63.

49. Soleimani Zadeh L. Danesh a, basri N, abaszadeh a, arab M. Assessment of high risk pregnancy in bam Mahdieh maternity hospital, 2001. J Shahrekord Univ Med Sci. 2004;6(2):67-73.

50. Modars Sh, Amidi Z. A survey on bacterial agents of asymptomatic bacteriuria in pregnant women in Tehran. Hakim Res J. 1998;2(1):23-6.

51. Sharemi H, Milani F, Zahiri Z, Zendedel M, Salamat F, Rafipour B, Javid Z. Comparison of pre-eclampsia risk factors regarding to its severity in pregnant women referred to Alzahra Hospital of Rasht, Iran, 2012. IJOGI. 2013;16(69):1-8

52. Sohrabi D, Ghanbari GM. A survey on risk factors and outcomes of women with preterm labor admitted to Valieasr hospital in Zanjan. J Urmia Nurs Midwifery Fac. 2011;9(2):84-9.

53. Amiri M, Lavasani Z, Norouzirad R, Najibpour R, Mohamadpour M, Nikpoor AR, Raeisi M, et al. Prevalence of Urinary Tract Infection Among Pregnant Women and its Complications in Their Newborns During the Birth in the Hospitals of Dezful City, Iran, 2012-2013. Iran Red Crescent Med J. 2015; 17(8):e26946.

54. Azhari S, Mousavi YM, Sojudi MM, EbrahimZadeh S. Accuracy of urine Dipslide to detect asymptomatic bacteriuria in pregnancy. IJOGI. 2011;15(3): 8-14

55. Sayehmiri K, Tavan H, Sayehmire F, Mohamadi I. Prevalence of Epilepsy in Iran Using Meta-Analysis and Systematic Review. zumsj. 2015;23(97):112-21.

56. Sayehmiri K, Tavan H. Systematic review and Meta- analysis methods prevalence of peptic ulcer in IRAN. J Govaresh. 2015;20(4):250-8.

57. Shamseer $L$, Moher D, Clarke M, Ghersi D, Liberati A, Petticrew M, et al. PRISMA-P Group. PRISMA-P Group. Preferred reporting items for systematic review and meta-analysis protocols (PRISMA-P) 2015:elaboration and explanation. BMJ. 2015;349:97647.

58. Richardson WS, Wilson MC, Nishikawa J, Hayward RS. The well-built clinical question:a key to evidence-based decisions. ACP J Club. 1995;123:A12-3.

59. Wells GA, Shea B, O'Connell D, Peterson J, Welch V, Losos M, et al. The Newcastle-Ottawa Scale (NOS) for assessing the quality of nonrandomized studies in meta-analyses. 2011. Available from:http://www.ohri.ca/programs/ clinical_epidemiology/oxford.asp. [cited 2012 Nov 25]

60. Guyatt GH, Oxman AD, Kunz R, Brozek J, Alonso-Coello P, Rind D, et al. GRADE guidelines 6. Rating the quality of evidence--imprecision. J Clin Epidemiol. 2011;64:1283-93. 21839614.

61. DerSimonian R, Laird N. Meta-analysis in clinical trials. Control Clin Trials. 1986;7:177-88. 
62. Ades AE, Lu G, Higgins JP. The interpretation of random-effects Metaanalysis in decision models. Med Decis Mak. 2005;25(6):646-54.

63. Al-Haddad AM. Urinary tract infection among pregnant women in AlMukalla district, Yemen. East Mediterr Health J. 2005:11:505-10.

64. Bandyopadhyay S, Thakur JS, Ray P. Kumar R.high prevalence of bacteriuria in pregnancy and its screening methods in North India. J Indian Med Assoc 2005;103:259-62.

65. Ghafari M, Baigi V, Cheraghi Z, Doosti-Irani A. The Prevalence of Asymptomatic Bacteriuria in Pregnant Iranian women:A Systematic Review and Meta-Analysis. Rosenfeld CS, ed. PLoS One. 2016;11(6):e0158031. https:// doi.org/10.1371/journal.pone.0158031.

66. Danesh Shahraki A, Pishva E, Mirbaha S, Arabzadeh A. The prevalence of asymptomatic Bacteruria in pregnant women with and without gestational diabetes. J Isfahan Med Sch. 2011;28(118):1214-22.

67. Mojahedi S, Baradaran H, Kordi M. Validity of urine analysis in diagnosis of asymptomatic bacteriuria in pregnancy. J Mashhad School Nurs Midwifery. 2000:4(11):32-8.

68. Daneshyar E, Mosavibahar SH, Alikhani MY. Association Between Asymptomatic Bacteriuria And Some emographic Variables in Pregnant Womenr Refered to Health Centers Affilited to Hamadan Univ Med Sci. sjimu. 2010;18(3):53-60.

69. Keshavarz M, Babaee G. Prevalence of Asymtomatic bacteriuria and drug sensitivity pattern inPregnant women. Med Sci J Islamic Azad Univ Mashhad. 2005;3(11):137-42.

70. Sujatha R, Nawani M. Prevalence of asymptomatic bacteriuria and its antibacterial susceptibility pattern among pregnant women attending the antenatal Clinic at Kanpur, India. J Clin Diagnostic Res. 2014;8(4):DC01-3.

71. Cunningham F, Leveno K, Hauth J, et al. Williams Obstetrics. 23rd ed. New York: McGraw-Hill Professional; 2009. p. 489-91.

72. Onu FA, Ajah LO, Ezeonu PO, Umeora OUJ, Ibekwe PC, Ajah MI. Profile and microbiological isolates of asymptomatic bacteriuria among pregnant women in Abakaliki, Nigeria. Infect Drug Resistance. 2015;8:231-5.

73. Imade PE, Izekor PE, Eghafona NO, Enabulele Ol, Ophori E. Asymptomatic bacteriuria among pregnant women. N Am J Med Sci. 2010;2(6):263-6 PubMed.

74. Thakur A, Baral R, Basnet P, Rai R, Agrawal A, Regmi MC, et al. Asymptomatic bacteriuria in pregnant women. JNMA. 2013;52(192):567-70.

75. Tadesse S, Kahsay T, Adhanom G, Kahsu G, Legese H, G/Wahid A, et al. Prevalence, antimicrobial susceptibility profile and predictors of asymptomatic bacteriuria among pregnant women in Adigrat General Hospital, Northern Ethiopia. BMC Res Notes. 2018;11(1):740.

76. Ronald AR, Pattullo AL. The natural history of urinary infection in adults. Med Clin North Am. 1991;75(2):299-312.

77. Ahmed F, Molla E, Eriso F. Prevalence and associated F infections among pregnant antenatal medical hospital, Borena zone. Merit Res J Med Sci. 2016;4(1):068-75

78. Foxman B. Epidemiology of urinary tract infections:incidence, morbidity, and economic costs. Am J Med. 2002;113(1):5-13.

79. Nicolle LE. Asymptomatic Bacteriuria and bacterial interference. Microbiol Spectr. 2015;3:1-25.

80. Hooton TM, Scholes D, Stapleton AE, Roberts PL, Winter C, Gupta K, et al. A prospective study of asymptomatic bacteriuria in sexually active young women. N Engl J Med. 2000;343:992-7.

81. Kunin $C M$, Deutscher R, Paquin A. Urinary tract infection in school children: an epidemiologic, clinical and laboratory study. Medicine (Baltimore). 1994; 43:91-130.

82. Mabbett AN, Ulett GC, Watts RE, Tree JJ, Totsika M, Ong CLY, Wood JM, Monaghan W, Looke DF, Nimmo GR, Svanborg C, Schembri MA. Virulence properties of asymptomatic bacteriuric Escherichia coli. Int J Med Microbiol. 2009;299:53.

83. Suriano F, Gallucci M, Flammia P, Musco S, Alcini A, Imbalzanot G, Dicuonzo G. Bacteriuria in patients with an orthoptic ileal neobladder:urinary tract infection or asymptomatic bacteriuria? Brit J Urol Internat. 2007;101:1576-9.

84. Hernandez JG, Sunden F, Connolly J, Svanborg C, Wullt B. Genetic control of the variable innate immune response to asymptomatic bacteriuria. PLoS One. 2011;6:e28289

85. Szweda $H$, Jóźwik M. Urinary tract infections during pregnancy - an updated overview. Dev Period Med. 2016;20(4):263-72.

86. De Wals P. Immunization strategies for the control of serogroup C meningococcal disease in developed countries. Expert Rev Vaccines. 2006;5(2):269-75.

87. Mathai E, Thomas RJ, Chandy S, Mathai M, Bergstrom S. Antimicrobials for the treatment of urinary tract infection in pregnancy: practices in southern India. Pharmacoepidemiol Drug Saf. 2004;13(9):645-52.

\section{Ready to submit your research? Choose BMC and benefit from:}

- fast, convenient online submission

- thorough peer review by experienced researchers in your field

- rapid publication on acceptance

- support for research data, including large and complex data types

- gold Open Access which fosters wider collaboration and increased citations

- maximum visibility for your research: over $100 \mathrm{M}$ website views per year

At BMC, research is always in progress.

Learn more biomedcentral.com/submissions 